\title{
Simultaneous palmar divergent dislocation of scaphoid and lunate concurrent with i psilateral humeroradial joint dislocation and humerus shaft fracture: report a rare case and add a new subtype
}

\section{Wan-wen Feng}

Lianyungang oriental hospital affiliated to Kangda college of Nanjing medical university

\section{Ya-yi Xia}

Lanzhou University Second Hospital

\section{Yue-peng Liu}

Lianyungang oriental hospital affiliated to Kangda college of Nanjing medical university

yuping liu ( $D$ ortholiuyp@163.com )

Tengzhou central hospital

\section{Case report}

Keywords: Scaphoid, Lunate, Palmar Divergent Dislocation, Humeroradial Joint Dislocation, Humerus Fracture

Posted Date: January 2nd, 2020

DOI: https://doi.org/10.21203/rs.2.19896/v1

License: (c) (1) This work is licensed under a Creative Commons Attribution 4.0 International License.

Read Full License 


\section{Abstract}

Background: Simultaneous palmar dislocation of scaphoid and lunate is a rare condition generally resulting from high-energy trauma and usually classified into two types. The literature has only reported nine patients with palmar divergent dislocation of scaphoid and lunate. Here we we present a case of accompanied ipsilateral humeroradial joint dislocation and open humeral shaft fracture caused by the same violence travelling from the wrist. Considering the emerging cases with their own characteristics, we suggest this severer injury be expanded into the third type of palmar dislocation of scaphoid and lunate. Case presentation: A 31-year-old male who fell from a three-storey building, with his right wrist directly hitting the ground, was admitted to the orthopaedic department of our hospital. Physical and radiographic examinations defined simultaneous palmar divergent dislocations of scaphoid and lunate concurrent with ipsilateral humeroradial joint dislocation and open humeral shaft fracture. After debridement and internal fixation for humerus and close reduction for humeroradial joint, the scaphoid and lunate were treated with open reduction and fixation by Kirschner's wires, and the palmar scapholunate ligament and anterior capsule were repaired through volar approach. After a 3-year followup, his humeral fracture healed without recurrent dislocations, collapse or avascular necrosis of scapholunate. Favorable hand function regained . Conclusions: Palmar divergent dislocation of scaphoid and lunate caused by considerable violence is rare and appropriate to urgent open reduction and fixation with Kirschner's wires and repair of the stronger palmar scapholunate ligament through single volar approach. When the residual violence continues to transmit to the upper extremity, ipsilateral dislocation or fracture may occur. We advise that this complex injury be classified into a third subtype of simultaneous palmar dislocation of scaphoid and lunate to avoid misdiagnosis.

\section{Background}

Simultaneous palmar dislocation of scaphoid and lunate generally resulting from high-energy trauma is extremely rare [1-9] and classified into two types depending on whether the scapholunate ligament is intact or not: 1) palmar dislocation as a unit and 2) palmar divergent dislocation [4-7]. Komura et al [7] reviewed the literature in 2011 and added a seventh case of simultaneous palmar divergent dislocations of scaphoid and lunate. Since then, the other two cases have been reported $[8,9]$, increasing the number of the patients with palmar divergent dislocations of scaphoid and lunate to nine [1-9]. Of the nine cases, one case presented with ipsilateral radial head fracture [1] and another with trapezium fracture [4]. But these two cases were all classified into the current system, regardless of their difference in concurrent fractures. In our case, ipsilateral humeroradial joint dislocation and open humeral shaft fracture were generated by the same high-energy trauma. Considering these fractures of different types, we suggest that they should be identified as a third type of simultaneous palmar dislocation of scaphoid and lunate. New classification system may raise our awareness of concomitant injures in ipsilateral upper extremity and avoid misdiagnosis.

\section{Case Presentation}


A 31-year-old male fell from a three-storey building and his right wrist hit the ground directly. He was admitted to the orthopaedic department of our hospital on October 25,2011 . An immediate physical examination found about a 4-cm open wound in his right upper arm with biceps torn and visible bleeding. His right elbow was immobile, as radial head showed a protruding deformity. He complained of severe swelling pain in his right wrist and a bony prominence on the palmar forearm. There were no signs of neurovascular disturbance. Radiographs of upper arm showed displacement fracture of the humeral shaft and radial dislocation of the humeroradial joint (Fig. 1A and B). Radiographs of the wrist revealed an abnormal increase in the scapholunate distance and an anterior dislocation of scaphoid and lunate (Fig. 1C and D). Computed tomography (CT) confirmed palmar divergent dislocation of scaphoid and lunate with a large gap between the two bones (Fig. 1E). About two hours after the injury, surgical intervention was pursued under general anesthesia. First, debridement and internal fixation with locking compression plate (LCP) were undertaken for the open humeral fracture, and then humeroradial joint was reduced manually (Fig. 2A). Finally, we used open reduction for scaphoid and lunate through palmar approach, because close reduction failed. Under fluoroscopy, two percutaneous Kirschner's wires were used to stabilize scaphoid and lunate (Fig. 2B and C). Subsequently, palmar scapholunate ligament and anterior capsule were repaired with absorbable threads through the same approach. A long arm plaster splint was applied to immobilize the wrist after surgery. At six weeks, the Kirschner's wires and plaster splint were removed, and the patient started intensive rehabilitation. The last follow-up at three years after injury noticed that our patient could live a normal life without any pain in his right upper limb.

Radiographs confirmed union of the humeral fracture, normal carpal alignment, no evidence of recurrent dislocations and no collapse of the scapholunate (Fig. 3A and B). Additionally, Magnetic resonance imaging (MRI) found no evidence of avascular necrosis of the scapholunate and the normal scapholunate interosseous distance (Fig. 3C). The right wrist retained nearly all its original functions. Range of motion: palmar flexion of $37^{\circ}$ (a $82 \%$ recovery according to that of the contralateral side, $45^{\circ}$ ), dorsiflexion of $80^{\circ}$ (equivalent to that of the contralateral side), ulnar deviation of $32^{\circ}$ (a $91 \%$ recovery according to that of the contralateral side, $35^{\circ}$ ), and radial deviation of $28^{\circ}$ (a $88 \%$ recovery according to that of the contralateral side, $32^{\circ}$ ). The grip strength of his injured hand (dominant) was comparable to his contralateral hand (Fig. 3D).

\section{Discussion And Conclusions}

As the patient fell to the ground on his right wrist, his hand outstretched and interosseous ligaments broke, resulting in the divergent dislocation of the scaphoid and the lunate. Not only that, the residual force travelled from the wrist to the upper part, leading to homolateral humeroradial joint dislocation and humeral fracture. Prior to this case report, one case with ipsilateral radial head fracture [1] and trapezium fracture was also described [4]. This type of injuries could be absorbed into a third subtype of palmar dislocation of scaphoid and lunate, just as Maisonneuve fracture of high fibular in Lauger-hansen classification of ankle fracture-dislocation [10]. With this new classification system, injury severity can be more accurately evaluated and coexisting injures can not be misdiagnosed. 
The optimal treatment of palmar dislocation of scaphoid and lunate remains unclear. In this case, we first fixed humeral shaft fracture and then reduced the dislocation, an effort that could prevent radial nerve from iatrogenic damage. Only one previous case had demonstrated postoperative avascular necrosis of lunate due to delayed diagnosis (33 days after injury) [3], and another treated with a proximal row carpectomy (PRC) (Table 1) [6]. Although PRC may eliminate avascular necrosis and avoid additional surgery, postoperative range of motion and grip strength just reach $50 \otimes 70 \%$ and $60 \otimes 90 \%$ of that of the healthy-side, respectively [11]. Therefore, except for special patients needing PCR, we recommend surgical repair as the first choice, especially for young people and manual workers [7].

Anatomic reduction can protect the scapholunate from further avascular damage and accelerate spontaneous revascularization [1-4, 6-9]. Closed reduction is technically difficult and cannot repair carpal interosseous ligaments. Moreover, repeated close reduction can damage the remaining soft tissue attachments of the scapholunate, which may contain important vessels needed to maintain blood supply to scaphoid and lunate $[3,9]$. Meanwhile, just a plaster cast is not enough to fix the scaphoid and lunate due to the ruptured interosseous ligaments and severe carpal instability $[3,4,7,8]$. As reported by Komura [7] and Idrissi [8], after closed reduction, palmar divergent dislocation of scaphoid and lunate still needed to be refixed because of its severe carpal instability. Furthermore, in our case, open reduction and fixation with Kirschner's wires also gained a favorable outcome.

Of previous reports, one case showed postoperative complication with flexion deformity of the scaphoid and a break in arc II of Gilula's line [7], one with avascular necrosis of the lunate [3], and another with scaphoid absorbed and cartilage height decreased and subchondral sclerosis and posttraumatic arthrosis [9]. These complications might be caused by additional dorsal incision and poor blood supply to scapholunate or no suturing in the stronger palmar scapholunate ligament [12]. In our case, with the palmar scapholunate ligament repaired through a single volar approach, the patient developed no avascular necrosis of carpal bones, indicating that the blood supply of scapholunate from surrounding soft tissue was rich enough for revascularization of scapholunate. Open reduction and ligament repair through only one palmar incision have more advantages: less invasive, smoother operation and maintenance of blood supply from soft tissues. Repairing the torn ligaments with a suture anchor can make operation simplify and offer non-space-consuming, dynamic, and permanent fixation, it has already been reported $[7,13]$.

In conclusion, we first reported a rare case of simultaneous palmar dislocation of scaphoid and lunate concurrent with the ipsilateral humeroradial joint dislocation and open humeral shaft fracture caused by the same traumatic event. We advise that this severer injury be divided into the third subtype of palmar dislocation of scaphoid and lunate. Once palmar dislocation of scaphoid and lunate diagnosed, it is recommended to perform in emergency open reduction and fixation with Kirschner's wires and repair of the stronger palmar scapholunate ligament through a single volar approach.

\section{Abbreviations}


AP: Anterior posterior; CT: Computed tomography; MRI: Magnetic resonance imaging; LCP: locking compression plate

\section{Declarations}

\section{Consent for publication}

Written informed consent was obtained from the patient for publication of this Case report and any accompanying images. A copy of the written consent is available for review by the Editor of this journal.

\section{Ethics approval and consent to participate}

Not applicable.

\section{Availability of data and materials}

All data generated or analyzed during this study are included in this

published article.

\section{Competing interests}

The authors declare that they have no competing interests.

\section{Funding}

The authors received no financial support for the research, authorship, and publication of this article.

\section{Authors' contributions}

FWW devised the technique, performed the surgery and was involved in data collection, case analysis and writing the manuscript. XYY participated in surgery, followed up the patient and case analysis. LYP followed up the patient and was involved in data collection and assisted in drafting the manuscript. LJK participated in the surgery, followed up the patient, and was involved in data collection and case analysis. LYP* participated in the surgery, followed up the patient, case analysis and and reviewed the article. All authors read and approved the final manuscript.

\section{Acknowledgements}

Not applicable.

\section{Authors' information}


${ }^{1}$ Department of Orthopaedic Surgery, Lianyungang Oriental Hospital Affiliated to Kangda College of Nanjing Medical University, Lianyungang, China.

${ }^{2}$ Department of Orthopaedic Surgery, Lanzhou University Second Hospital, Lanzhou, China.

${ }^{3}$ Department of Orthopaedic Surgery, Tengzhou Central People's Hospital, Tengzhou, China.

\section{References}

1. Gordon SL. Scaphoid and lunate dislocation. Report of a case in a patient with peripheral neuropathy. J Bone Joint Surg Am.1972; 54:1769-72

2. Somford MP, Sturm MF, Vroemen JP.Reconstruction of isolated scaphoid dislocation with carpal dissociation, associated with a carpal anomaly. Strategies Trauma Limb Reconstr. 2010 ;5(2):105-110. 3. Coll GA.Palmar dislocation of scaphoid and lunate. J Hand Surg Am.1987; 12:476-480.

4. Baulot E, Perez A, Hallonet D, et al. Scaphoid and lunate palmar divergent dislocation. Apropos of a case. Rev Chir Orthop Reparatrice Appar Mot. 1997; 83: 265-9.

5.Kang HJ,Shim DJ,Hahn SB,Kang ES.Palmar divergent dislocation of scaphoid and lunate. Yonsei Med J.2003; 44:1091-3.

6. Domeshek LF, Harenberg PS, Rineer CA,et al. Total scapholunate dislocation with complete scaphoid extrusion: case report. J Hand Surg Am.2010; 35:69-71.

7. Komura S, Yokoi T, Suzuki Y. Palmar-divergent dislocation of scaphoid and lunate. J Orthop Traumatol.2011; 12: 65-8.

8. Idrissi KK, Galiua F.Palmar dislocation of scaphoid and lunate. Clin Pract.2011; 1:e87.

9. Phan KH, Azimi HJ, Franko Ol, Abrams RA. Scaphoid and Lunate Dislocation with Complete SoftTissue Avulsion: A Case Report. JBJS Case Connect. 2016; 6: e58. doi: 10.2106/JBJS.CC.15.00112. 10. Pankovich AM. Maisonneuve fracture of the fibula. J Bone Joint Surg Am. 1976 ; 58: 337-42.

11. Edouard P, Vernay D, Martin S, et al. Proximal row carpectomy: Is early postoperative mobilisation the right rehabilitation protocol? Orthop Traumatol Surg Res.2010; 96: 513-20.

12. Short WH, Werner FW,Green JK,et al. Biomechanical evaluation of the ligamentous stabilizers of scaphoid and lunate: part III. J Hand Surg Am. 2007; 32: 297-309.

13. Kang L, Ek ET, Wei MT, et al. Biomechanical Analysis of Scapholunate Ligament Repair Techniques. J Hand Surg Am. 2015; 40: 1534-9.

\section{Table}

Due to technical limitations, table 1 is only available as a download in the supplemental files section.

\section{Figures}




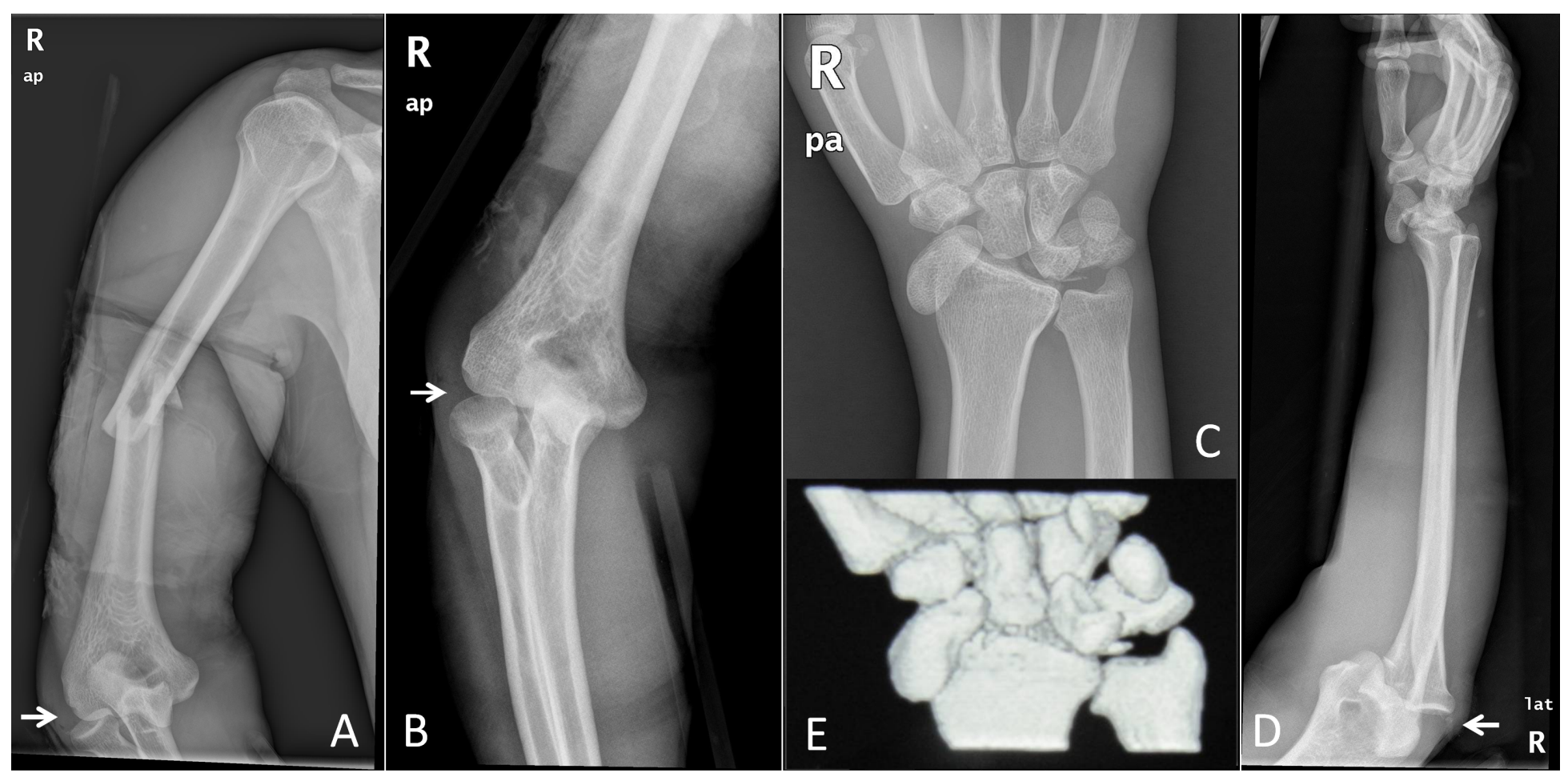

Figure 2

Preoperative radiographs. A Anteroposterior radiograph of the upper arm showed the humeral shaft fracture; B Anteroposterior radiograph of the elbow revealed radial dislocation of humeroradial joint (arrows); C, D Anterioposterior radiograph and lateral radiographs of the forearm both showed palmar divergent dislocation of the scapholunate; E CT scan of the wrist also verified palmar divergent dislocation of the scaphoid and the lunate. The arrows indicate radial dislocation of humeroradial joint 


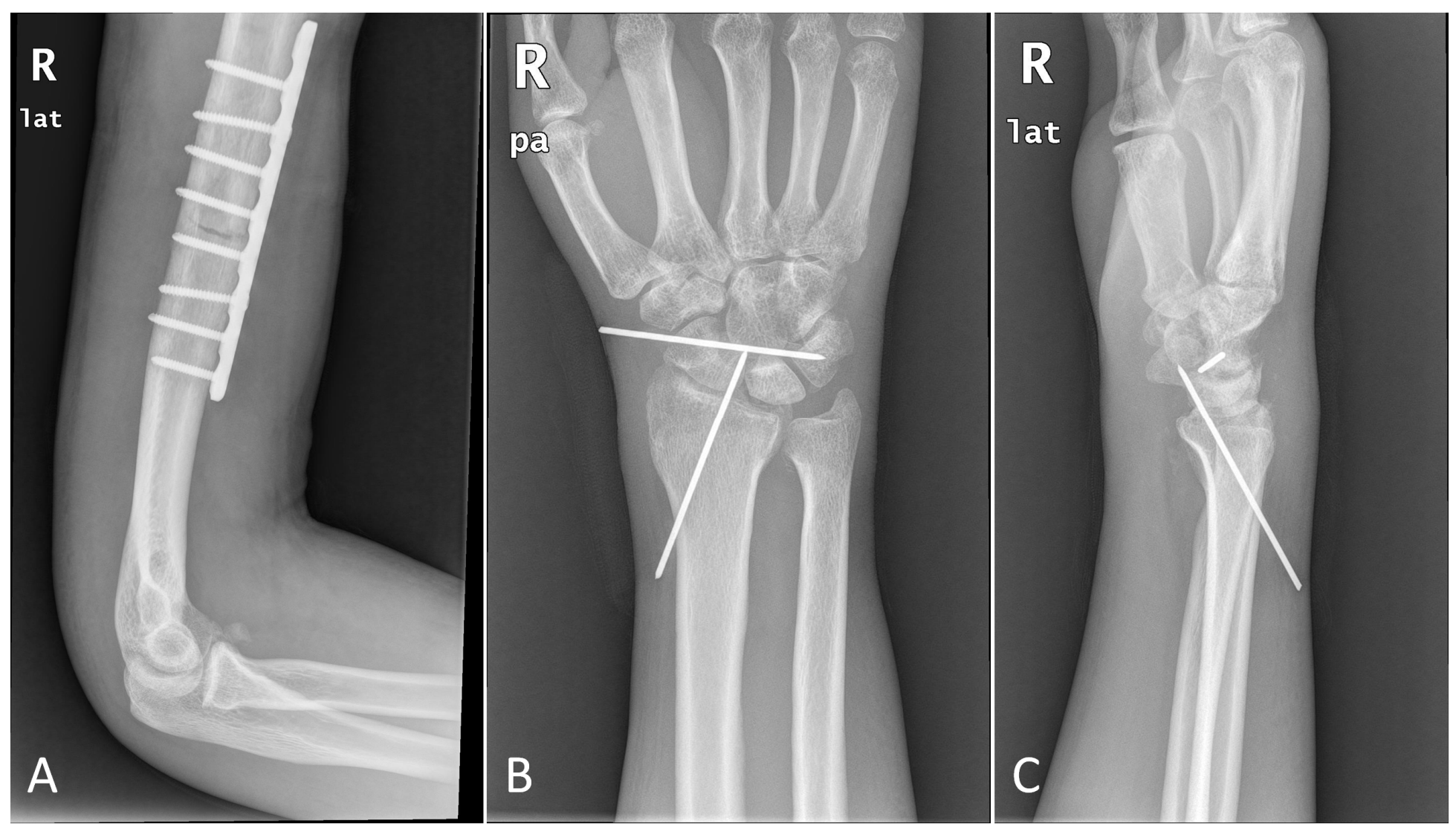

\section{Figure 3}

Postoperative radiograms. A Lateral radiogram of the upper arm showed reduction fixation of the humeral shaft fracture and reduction of the humeroradial joint; B, C Reduction fixation of the palmar divergent dislocation of the scapholunate were visible in anteroposterior radiogram of the wrist and lateral radiogram of the wrist 


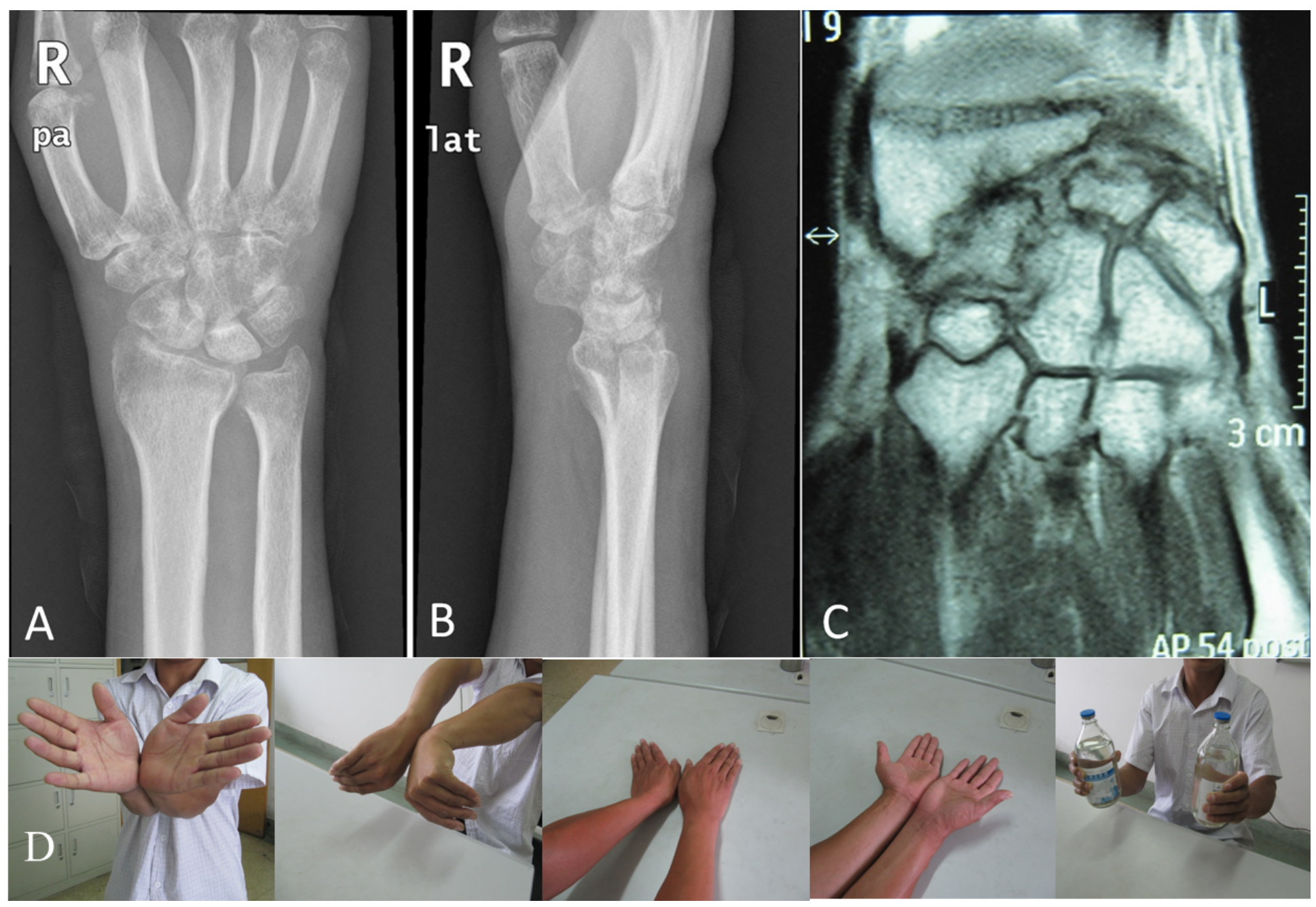

\section{Figure 6}

The wrist radiographs, MR image and function display at the final follow-up. A, B A normal carpal alignment, no evidence of recurrent dislocations and no collapse of the scapholunate were shown in anteroposterior radiograph of the wrist and lateral radiograph of the wrist; C MR image of the wrist verified no evidence of avascular necrosis of the scapholunate; D Good function of the wrist was displayed

\section{Supplementary Files}

This is a list of supplementary files associated with this preprint. Click to download.

- CAREchecklistEnglish2013.pdf

- Consentform.emergencysurgery.tif

- Consentform.emergencysurgery.tif

- CAREchecklistEnglish2013.pdf

- Table1.Summaryofpreviouspatients.xlsx 
- Table1.Summaryofpreviouspatients.xIsx 